\title{
Do wrist guards have the potential to protect against wrist injuries in bicycling, micro scooter riding, and monkey bar play?
}

\author{
E Cassell, K Ashby, A Gunatilaka, A Clapperton
}

Objective: To test the potential of wrist guards to prevent wrist injury in recreational activities that require good grip strength and hand dexterity.

Methods: Forty eight children aged 5-8 years from one Melbourne school volunteered for tests chosen or devised following a review of the literature on the effects of orthoses use and hand function.

Results: Wrist guard wearing significantly degraded grip strength, bicycle steering, and performance on a monkey bar. Micro scooter steering was not significantly affected by their use.

Conclusion: Wrist guards designed for skating are not recommended for bicycle riding and playing on climbing apparatus, and their suitability for scooter riding requires further investigation.

W ist guards are designed to protect the wrist against injury in a fall onto an outstretched hand. ${ }^{1}$ Evidence supports their effectiveness in inline skating and snowboarding, ${ }^{1-4}$ although wearing rates in these sports are generally reported to be low. ${ }^{15-8}$

Injury surveillance hospital data from Victoria, Australia indicate that wrist and distal forearm fractures in children are more frequent in bicycle riding and monkey bar play than in inline skating, an activity for which wrist guards are recommended (VISAR, unpublished data). In 2002/03, there were at least 196 forearm and wrist fractures related to inline skating treated in Victorian hospitals compared with 78 in micro scooter riding, 294 in bicycle riding, and over 600 in monkey bar play (VISAR, unpublished data). Research indicates that the trauma mechanism for wrist and distal forearm injuries (fall on an outstretched arm) is similar for all these activities. ${ }^{9-11}$ Reaching out the hand to break a fall, to protect more vulnerable areas of the body, appears to be a reflexive action in humans. ${ }^{12}{ }^{13}$ Currently there is no evidence of the potential effectiveness of wrist guards in recreational activities that require grip and wrist dexterity.

A typical micro scooter has a narrow metal base with two small, low friction wheels and a handlebar mounted on a collapsible stem. A monkey bar, also known as a horizontal ladder or jungle gym, is a type of playground equipment that consists of two parallel horizontal bars supported at a height, and traversed by rungs.

\section{MAIN OUTCOME MEASURES}

The main outcome measures were differences in the performance with and without wrist guards in static tests that measured maximum grip strength and maximum torque exerted on bicycle and scooter handlebars, and in dynamic tests that measured the number of deviations from a marked figure 8 track by the micro scooter or bicycle when ridden, and the number of rungs traversed along a monkey bar before grip was lost.

The null hypothesis was that there is no statistical difference in results of hand function tests between wrist guard wearers and non-wearers.

\section{METHODS}

Forty eight children aged 5-8 years volunteered for the study with parental consent. Children were from one government school situated in a suburb of Melbourne. Exclusions included non-bike riders and children who had an existing or previous severe hand injury. Approval was obtained from 
Table 1 Mean, standard deviation (SD), standardised mean differences with and without wrist guards ( $\mathrm{z}$ scores), and Wilcoxon test significance values in grip strength test and monkey bar test for all subjects and by sex, age, and hand length

\begin{tabular}{|c|c|c|c|c|c|c|}
\hline & \multicolumn{3}{|l|}{ Grip strength } & \multicolumn{3}{|c|}{ Traversing monkey bar } \\
\hline & $\begin{array}{l}\text { With WG, } \\
\text { mean (SD) }\end{array}$ & $\begin{array}{l}\text { Without WG, } \\
\text { mean (SD) }\end{array}$ & $z$ (p value) & $\begin{array}{l}\text { With WG, } \\
\text { mean (SD) }\end{array}$ & $\begin{array}{l}\text { Without WG, } \\
\text { mean (SD) }\end{array}$ & $z$ (p value) \\
\hline All subjects $(n=48)$ & $7.43(2.46)$ & $9.68(3.31)$ & $-5.70 *(<0.001)$ & $3.89(3.43)$ & $10.51(3.06)$ & $-5.65^{*}(<0.001)$ \\
\hline \multicolumn{7}{|l|}{ Sex } \\
\hline Male $(n=25)$ & $7.49(2.84)$ & $10.11(3.86)$ & $-4.21^{*}(<0.001)$ & $4.21(3.75)$ & 9.88 (3.67) & $-4.02 *(<0.001)$ \\
\hline Female $(n=23)$ & $7.36(2.03)$ & $9.22(2.58)$ & $-3.91 *(<0.001)$ & $3.52(3.06)$ & $11.27(1.96)$ & $-4.02 *(<0.001)$ \\
\hline \multicolumn{7}{|l|}{ Age (years) } \\
\hline $5 \& 6(n=20)$ & $6.00(1,81)$ & 7.70 (1.83) & $-3.51 *(<0.001)$ & $1.88(1.19)$ & $9.37(4.06)$ & $-3.83^{*}(<0.001)$ \\
\hline $7(n=16)$ & $7.67(2.23)$ & $10.27(2.95)$ & $-3.52^{*}(<0.001)$ & $4.47(3.18)$ & $11.67(0.93)$ & $-3.18^{*}(0.001)$ \\
\hline $8(n=12)$ & $9.50(2.24)$ & $12.19(3.81)$ & $-2.82^{*}(0.005)$ & $6.50(4.30)$ & $11.08(2.11)$ & $-2.80 *(0.005)$ \\
\hline \multicolumn{7}{|l|}{ Hand length $(\mathrm{cm})$} \\
\hline $11.5-13.9(n=11)$ & $5.45(1.78)$ & $7.21(2.08)$ & $-2.94^{*}(0.003)$ & $2.12(1.75)$ & $8.79(4.56)$ & $-2.95 *(0.003)$ \\
\hline $14.0-14.9(n=19)$ & $7.37(2.19)$ & $9.42(2.79)$ & $-3.46^{*}(0.001)$ & $2.93(2.15)$ & $11.00(2.50)$ & $-3.73^{*}(<0.001)$ \\
\hline $15.0-17.5(n=18)$ & $8.70(2.37)$ & $11.46(3.48)$ & $-3.60^{*}(<0.001)$ & $6.10(4.27)$ & $11.13(1.97)$ & $-3.18^{*}(0.001)$ \\
\hline
\end{tabular}

Bold formatting indicates a statistically significant difference between the wearing and non-wearing of wrist guard conditions.

*Based on negative rank indicates lower scores on tests with wrist guards; in the above two tests a lower score indicates performance degradation.

the Monash University Human Research Ethics Committee and the Department of Education. Subjects were not blinded to the objectives of the study because the education department and ethics committee required full disclosure of the study objectives to parents and children. Outcome assessors were not blinded because it was not practicable. The order in which subjects participated in tests and performed a given test with or without wrist guards was randomised by coin flip in advance of the test day. Only one rater per test was used. An electronic sensing device aided the rater counting deviations from the figure 8 track.

\section{Safety measures}

Subjects wore helmets during the bicycle steering test. Elbow pads, kneepads, and helmets were worn during scooter steering tests. Appropriately sized bicycles were supplied and subjects could opt to use training wheels. A specially modified (three wheeled) micro scooter was supplied. A fall arrest harness was used during monkey bar tests.

\section{Data collection}

Data collected via parental questionnaire included age, school grade, sex, and handedness. Subjects' height, weight, and dominant hand length were recorded.
Subjects performed the series of tests with and without wrist guards. Each test was performed three times and the mean score recorded.

\section{Description of tests}

Tests were chosen or devised following a review of the literature on the effects of orthoses use and hand function. ${ }^{14-19}$ The hand dynamometer was reported to give an accurate measure of grip strength. ${ }^{18-20}$ The handlebar torque test was developed in collaboration with the Department of Civil Engineering at Monash University. The track tests were developed taking cognizance of expert opinion that hand function tests should measure the dynamic quality of function and use tasks representative of everyday functional activity. ${ }^{16}$

\section{Grip strength test}

A Jamar hand dynamometer (Sammons Preston Inc, Bolingbrook, IL, USA) measured grip strength of the dominant hand in kilogram force ( $1 \mathrm{kgf}=9.8$ newtons) with and without wrist guards.

\section{Handlebar torque tests}

A scooter and a bicycle instrumented with strain-gauge sensors measured the maximum torque (in newton meters)

Table 2 Mean, standard deviation (SD), standardised mean differences with and without wrist guards ( $z$ scores) and Wilcoxon test significance values in bicycle torque test and steering test for all subjects and by sex, age, and hand length

\begin{tabular}{|c|c|c|c|c|c|c|}
\hline & \multicolumn{3}{|c|}{ Bicycle handlebar torque test } & \multicolumn{3}{|c|}{ Bicycle steering track test } \\
\hline & $\begin{array}{l}\text { With WG, } \\
\text { mean (SD) }\end{array}$ & $\begin{array}{l}\text { Without WG, } \\
\text { mean (SD) }\end{array}$ & $z$ (p value) & $\begin{array}{l}\text { With WG, } \\
\text { mean (SD) }\end{array}$ & $\begin{array}{l}\text { Without WG, } \\
\text { mean (SD) }\end{array}$ & $z$ (p value) \\
\hline All subjects $(n=48)$ & $118.0(69.7)$ & $123.3(72.2)$ & $-0.65^{\star}(0.519)$ & $1.28(1.06)$ & $0.94(1.00)$ & $-2.47 \dagger(0.013)$ \\
\hline \multicolumn{7}{|l|}{ Sex } \\
\hline Male $(n=25)$ & $143.3(74.5)$ & $149.7(77.0)$ & $-0.31 *(0.757)$ & $1.35(1.09)$ & $1.05(1.07)$ & $-1.51+(0.131)$ \\
\hline Female $(n=23)$ & $90.51(53.0)$ & $94.5(55.0)$ & $-0.63^{*}(0.527)$ & $1.20(1.04)$ & $0.82(0.91)$ & $-2.13+(0.033)$ \\
\hline \multicolumn{7}{|l|}{ Age (years) } \\
\hline $5 \& 6(n=20)$ & $124.2(72.3)$ & $122.3(59.7)$ & $-0.22 \dagger(0.823)$ & $1.56(1.29)$ & $0.95(1.24)$ & $-2.37 \dagger(0.018)$ \\
\hline $7(n=16)$ & $92.9(62.4)$ & $99.6(68.3)$ & $-0.23^{*}(0.820)$ & $0.90(0.81)$ & $0.96(0.96)$ & $-1.17^{*}(0.241)$ \\
\hline $8(n=12)$ & $141.1(69.7)$ & $156.4(88.2)$ & $-1.96 *(0.050)$ & $1.33(0.84)$ & $0.92(0.59)$ & $-2.02+(0.043)$ \\
\hline \multicolumn{7}{|l|}{ Hand length $(\mathrm{cm})$} \\
\hline $11.5-13.9(n=11)$ & $105.2(90.7)$ & $103.6(79.3)$ & $-0.27+(0.790)$ & $1.39(1.6)$ & $0.79(1.29)$ & $-2.12 \dagger(0.034)$ \\
\hline $14.0-14.9(n=19)$ & $110.4(52.6)$ & 105.7 (43.5) & $-0.77+(0.445)$ & $1.04(0.91)$ & $0.89(0.96)$ & $-0.82+(0.410)$ \\
\hline $15.0-17.5(n=18)$ & $133.9(72.3)$ & $153.8(84.6)$ & $-2.30 *(0.022)$ & $1.44(0.72)$ & $1.09(0.86)$ & $-1.34 \dagger(0.181)$ \\
\hline
\end{tabular}

Bold formatting indicates a statistically significant difference between the wearing and non-wearing of wrist guard conditions.

*Based on negative rank indicates lower scores on tests with wrist guards and tbased on positive rank indicates higher scores on tests with wrist guards; in the bicycle handlebar torque test a lower score indicates performance degradation, in the bicycle steering track test a lower score indicates improved performance. 
Table 3 Mean, standard deviation (SD), standardised mean differences with and without wrist guards ( $z$ scores) and Wilcoxon test significance values in scooter torque test and steering test for all subjects and by sex, age, and hand length

\begin{tabular}{|c|c|c|c|c|c|c|}
\hline & \multicolumn{3}{|c|}{ Scooter handlebar torque test } & \multicolumn{3}{|c|}{ Scooter steering track test } \\
\hline & $\begin{array}{l}\text { With WG, } \\
\text { mean (SD) }\end{array}$ & $\begin{array}{l}\text { Without WG, } \\
\text { mean (SD) }\end{array}$ & $z$ (p value) & $\begin{array}{l}\text { With WG, } \\
\text { mean (SD) }\end{array}$ & $\begin{array}{l}\text { Without WG, } \\
\text { mean (SD) }\end{array}$ & $z$ (p value) \\
\hline All subjects $(n=48)$ & $177.2(140.8)$ & $174.7(142.5)$ & $-0.25+(0.806)$ & $0.51(0.68)$ & $0.50(0.51)$ & $-0.24^{*}(0.809)$ \\
\hline \multicolumn{7}{|l|}{ Sex } \\
\hline Male $(n=25)$ & $220.8(141.7)$ & $215.5(142.3)$ & $-0.01+(0.989)$ & $0.51(0.75)$ & $0.56(0.55)$ & $-0.70 *(0.487)$ \\
\hline Female $(n=23)$ & $130.0(126.3)$ & $130.5(131.8)$ & $-0.70 \dagger(0.484)$ & $0.52(0.61)$ & $0.44(0.48)$ & $-0.40+(0.690)$ \\
\hline \multicolumn{7}{|l|}{ Age (years) } \\
\hline $5 \& 6$ old $(n=20)$ & $193.0(148.1)$ & $195.6(140.3)$ & $-0.49 *(0.627)$ & $0.70(0.78)$ & $0.58(0.56)$ & $-0.64 \dagger(0.524)$ \\
\hline 7 old $(n=16)$ & $134.0(112.2)$ & $130.2(133.9)$ & $-1.50+(0.134)$ & $0.44(0.66)$ & $0.46(0.54)$ & $-0.26^{*}(0.794)$ \\
\hline 8 old $(n=12)$ & $208.7(159.4)$ & $199.4(154.7)$ & $-0.08+(0.937)$ & $0.21(0.46)$ & $0.44(0.41)$ & $-1.04^{*}(0.298)$ \\
\hline \multicolumn{7}{|l|}{ Hand length $(\mathrm{cm})$} \\
\hline $11.5-13.9(n=11)$ & $149.6(167.6)$ & $166.4(156.6)$ & $-1.42^{*}(0.155)$ & $0.39(0.53)$ & $0.52(0.48)$ & $-0.88^{*}(0.380)$ \\
\hline $14.0-14.9(n=19)$ & $166.7(123.9)$ & $148.9(128.5)$ & $-1.37 \dagger(0.171)$ & $0.63(0.84)$ & $0.59(0.58)$ & $-0.21^{*}(0.831)$ \\
\hline $15.0-17.5(n=18)$ & $205.4(143.5)$ & $207.1(149.3)$ & $-0.20 *(0.845)$ & $0.46(0.60)$ & $0.41(0.48)$ & $-0.45+(0.650)$ \\
\hline
\end{tabular}

*Based on negative rank indicates lower scores on tests with wrist guards and tbased on positive rank indicates higher scores on tests with wrist guards; in the scooter handlebar torque test a lower score indicates performance degradation, in the scooter steering track test a lower score indicates improved performance.

exerted on the handlebars by subjects when they turned the handlebar to the left and to the right, with and without wrist guards.

\section{Monkey bar test}

Participants traversed a $3.7 \mathrm{~m}$ long, straight, horizontal monkey bar, which had 11 horizontal rungs of $3.8 \mathrm{~cm}$ diameter at $12.5 \mathrm{~cm}$ separation. The number of rungs the subject could traverse, with and without wrist guards, before losing grip was recorded.

\section{Bicycle (scooter) track test}

Subjects rode a bicycle (scooter) at moderate speed along a $38 \mathrm{~m}$ figure 8 shaped track marked on an asphalt surface with and without wrist guards. The track was $60 \mathrm{~cm}$ wide. The number of deviations from the track was recorded.

\section{Statistical analysis}

The study used a repeated measures design. Subjects acted as their own controls. SPSS version 11.5 statistical analysis software was used to analyze data (SPSS Inc, Chicago, IL, USA).

Because data were not normally distributed, the Wilcoxon matched pairs signed ranks test was used to detect if the mean differences in the measures were significant.

\section{RESULTS}

The mean and standard deviation (SD) with and without wrist guards, the standardized mean differences in performance ( $\mathrm{z}$ scores) with and without wrist guards, and Wilcoxon test significance values for each of the tests are summarized in tables $1-3$.

In summary, for all subjects when wrist guards were worn:

- the maximum grip strength exerted by the dominant hand decreased significantly $(\mathrm{z}=-5.70, \mathrm{p}<0.001)$ (table $\mathrm{l}$ )

- there were significant degradations in subjects' performance traversing the monkey bar $(\mathrm{z}=-5.65, \mathrm{p}<0.001)$ (table 1)

- there was significant degradation in bicycle steering performance in the bicycle track test $(\mathrm{z}=-2.47$, $\mathrm{p}=0.013$ ), but no significant difference was apparent in the bicycle torque test (table 2).

There was no significant difference in micro scooter steering performance when wrist guards were worn or not worn, as measured by both the static torque test and the figure 8 track (table 3 ).

\section{DISCUSSION}

This is the first study to investigate the important question of whether wrist guards have the potential to protect against wrist injuries for recreational activities that require grip, without producing other unwanted effects. The adverse results of grip strength and monkey bar tests indicate that the wearing of wrist guards could potentially increase fall related injuries to non-protected body sites if adopted by children for play on monkey bars and other climbing apparatus.

Similarly wearing of wrist guards significantly increased the number of steering deviations off the test track when children rode the bicycle. This appeared to be related to subjects slowing down considerably when riding with wrist guards and losing their line of steering. This effect may have been related to loss of dexterity.

Wrist guards did not appear to adversely affect steering of the micro scooter over a test track. However, widespread adoption of wrist guards for scootering is not recommended until results are confirmed on a more challenging track.

There was no significant difference overall in the maximum torque exerted by subjects in the bicycle and micro scooter handlebar static tests performed with or without wrist guards. The difference between the results of static and dynamic bicycle steering tests suggest that the static test may not give a reliable indication of real life bicycle steering performance, perhaps because of the complications of having the brakes on the bike handlebars.

\section{LIMITATIONS OF THE STUDY}

The figure 8 track may not have been sufficiently challenging to test steering ability in micro scooter riding. Also, the riding speed was not controlled in the steering tests. Some subjects were observed to slow down considerably when riding the bicycle wearing wrist guards. Because steering accuracy is likely to depend in part on the speed of travel, the time taken to complete the test course should be measured in any subsequent study.

There were published norms for only the Jamar hand dynamometer test but the population on which they are based (US children resident in Milwaukee) was very different from our sample, which was 50\% Asian background. 


\section{Key points}

- Grip strength is reduced when wrist guards are worn.

- Wearing of wrist guards significantly degrades children's ability to traverse a monkey bar and the accurate steering of a bicycle, but not a micro scooter.

- Wrist guards designed for skating are not recommended for bicycle riding and playing on climbing apparatus, and their suitability for scooter riding requires further investigation.

The small sample size used in this study did not facilitate analysis of interaction effects between sex, age, and hand length.

\section{FUTURE DIRECTIONS}

None of the wrist guards marked as "small" were a good fit for children with small hands and the volar plates extended well up the palm beyond the distal palmar crease.

These design faults adversely affected the grip that smaller children had on the monkey bar rungs, although their grip was generally tenuous without the wrist guards because of the diameter of the rungs $(3.8 \mathrm{~cm})$. Design deficiencies in wrist guards have been noted previously. ${ }^{21}$ Overseas researchers have improved the design of wrist guards for snowboarding, including the use of a new polyester elastomer, which provides better impact absorption and is more flexible, allowing the user to retain the maximum mobility of the arm.

Research is required to investigate if this material and innovative design features could be combined to develop a wrist guard that is more appropriate to recreational activities that demand good grip and dexterous use of the wrist.

\section{ACKNOWLEDGEMENTS}

This study was supported by the Victorian Health Promotion Foundation and the Monash University Accident Research Centre Strategic Development Program. The authors acknowledge the support of Mr Stuart Newstead who advised on statistical analysis, and of the Principal, staff, and students of Glen Waverley South Primary School.

\section{Authors' affiliations}

E Cassell, K Ashby, A Gunatilaka, A Clapperton, Monash University Accident Research Centre, Melbourne, Australia
The authors have no competing interests with regard to this research.

Ethics Approval was obtained from the Monash University Human Research Ethics Committee and the Department of Education of Victoria.

Correspondence to: Ms E Cassell, Accident Research Centre, Building 70, Monash University, Victoria 3800, Australia; erin.cassell@general. monash.edu.au

Accepted 19 February 2005

\section{REFERENCES}

1 Schieber R, Branche-Doresy C, Ryan G, et al. Risk factors for injuries from inline skating and the effectiveness of safety gear. N Engl J Med 1996;335:1630-5.

2 Lewis L, West O, Standevan J, et al. Do wrist guards protect against fractures? Ann Emerg Med 1997;29:766-9.

3 Machold W, Kwasny O, Eisenhardt P, et al. Reduction of severe wrist injuries in snowboarding by an optimized wrist protection device: A prospective randomized trial. J Trauma 2002;52:517-20.

4 Staebler M, Moore D, Akelman E, et al. The effect of wrist guards on bone strain in the distal forearm. Am J Sports Med 1999;27:500-6.

5 Beirness DJ, Foss RD, Desmond KJ. Use of protective equipment by in-line skaters: an observational study. Inj Prev 2001;7:51-5.

6 Sherker S, Cassell E. Personal protective equipment use by in-line skaters in Victoria. Aust N Z J Public Health $2001 ; 25: 179-84$.

7 O'Neill DF. Wrist injuries in guarded versus unguarded first time snowboarders. Clin Orthop 2003;409:91-5.

8 Made C, Elmqvist L-G. A 10-year study of snowboard injuries in Lapland Sweden. Scand J Med Sci Sports 2004; 14:128-33.

9 Chapman S, Webber C, O'Meara M. Scooter injuries in children. J Paediatr Child Health $2001 ; 37: 567-70$.

10 Schalamon J, Sarkola T, Nietosvaara Y. Injuries in children associated with the use of nonmotorized scooters. J Pediatr Surg 2003;38:1612-15.

11 Adeboye K, Armstrong L. Pattern and severity of injuries in micro-scooter related accidents. Emerg Med J 2002; 19:571-2.

12 Cummings S, Nevitt M. A hypothesis: the causes of hip fractures. J Gerontol 1989:44:M107-11

13 Hsiao E, Robinovitch S. Common protective movements govern unexpected falls from standing height. J Biomech 1998;31:1-9.

14 Stern EB. Grip strength and finger dexterity across five styles of commercial wrist orthoses. Am J Occup Ther 1996;50:32-8.

15 Pagnotta D, Baron M, Korner-Bitensky N. The effect of a static wrist orthosis on hand function in individuals with Rheumatoid Arthritis. J Rheumatol 1998;25:879-85.

16 McPhee S. Functional hand evaluations: A review. Am J Occup Ther 1987;41:158-63

17 LaStayo $\mathbf{P}$, Hartzel J. Dynamic versus static grip strength: How grip strength changes when the wrist is moved, and why dynamic grip strength may be a more functional measurement. J Hand Ther 1999;12:212-18.

18 Kellor M, Frost J, Silberberg N, et al. Hand strength and dexterity. Am J Occup Ther 1971;25:77-83

19 Mathiowetz V, Kashman N, Volland G, et al. Grip and pinch strength: normative data for adults. Arch Phys Med Rehabil 1985;66:69-74.

20 Mathiowetz V, Weber K, Volland G, et al. Reliability and validity of hand strength evaluation. J Hand Surg 1984:9:222-6.

21 Hwang IK, Kim KJ. Shock absorbing effects of various padding conditions in improving efficacy of wrist guards. J Sports Sci Med 2004;3:23-9. 